\title{
Mechanism behind columnar pattern formation during directional quenching-induced phase separation
}

\author{
Tsuyoshi Tsukada and Rei Kurita \\ Department of Physics, Tokyo Metropolitan University, 1-1 Minamioosawa, Hachiouji-shi, Tokyo 192-0397, Japan
}

(Received 19 December 2019; accepted 13 March 2020; published 31 March 2020)

\begin{abstract}
It is known that there is a universality behind pattern formation during phase separation induced by homogeneous quenching. However, this universality is broken when quenching is carried out in an inhomogeneous manner. An example is directional quenching, where a columnar or lamellar pattern may be formed depending on the speed of the quenching front. Despite the wide industrial and technological interest in pattern formation, the mechanism behind the formation of these columnar patterns is not yet understood. Here we investigated the dynamics of pattern formation induced by directional quenching using numerical simulations. We found that the interface of the first layer that is formed fluctuates, and a columnar pattern is formed when this fluctuation grows. In addition, we reveal that the effective confinement effect induced by the quenching front plays an important role in determining the nature of the fluctuations at the interface.
\end{abstract}

DOI: 10.1103/PhysRevResearch.2.013382

\section{INTRODUCTION}

Phase separation may occur when a well-mixed binary system such as a metal alloy or liquid mixture is homogeneously quenched. As the system phase separates, a droplet pattern or a bicontinuous pattern is formed [1-11]. Since patterns are directly related to many physical properties, control over pattern formation has been the focus of many studies [4]. But there is a universality behind pattern formation during homogeneous phase separation (the so-called model B [12]); thus, other quenching methods are required to control and diversify the patterns which are formed. One such method is inducing phase separation in templated quenching systems [13-20], where phase separation is affected by the boundaries of a volume due to wetting effects. Investigations which apply asymmetric affinity to walls [13-16], Janus particles [17-19], and templated colloids [20] have also been carried out. The effect of confinement may also play an important role for phase separation in porous media [21]. However, although these methods enable a degree of control over pattern formation, the effect of the actual walls or particles used may have a non-negligible impact on physical properties. Alternative, indirect means to control phase separation would be invaluable.

Some decades ago, Furukawa proposed an alternative method for controlling pattern formation which do not affect the properties of a material by themselves [22]. Region where phase separation occurs, or the quenched region, spreads in one direction, which is called a directional quenching method.

Published by the American Physical Society under the terms of the Creative Commons Attribution 4.0 International license. Further distribution of this work must maintain attribution to the author(s) and the published article's title, journal citation, and DOI.
The pattern formed depends on the migration speed of the quenching front $V$ [22-24]. When $V$ is fast, a droplet pattern is formed just like for homogeneous quenching. When $V$ is comparable to the speed at which phase separation takes place, a lamellar pattern forms which is parallel to the quenching front. Finally, when $V$ is much slower, these layers can even be aligned perpendicular to the quenching front. This final phase is called a columnar pattern.

Recently, there have been numerous studies of directional quenching [20,25-33]. In previous work, we reported formation of a large length-scale periodic pattern using a radial quench [27]; we also showed that the width of the lamellae can be controlled using $V$ in a three-dimensional numerical simulation with parameters which replicated realistic experimental conditions [32]. Furthermore, we investigated the effect of confinement on phase separation induced by radial quenching [33]. Due to an inhomogeneous temperature field, the flux of each component of the mixture is anisotropic; this anisotropic flow can be suppressed by confinement, leading to a topological transition from three-dimensional to twodimensional patterns. Directional quenching may also be seen in experimental systems. For example, when a system with particle inclusions is quenched, phase separation is seen to occur near the particles first since they have a greater heat conductivity, creating a temperature front $[29,30]$.

Although directional quenching has been addressed in previous work, the transition from a lamellar pattern to a columnar pattern, one of the most notable, fundamental transitions seen in directional quenching is yet to be properly explained. We stress that an understanding of columnar pattern formation is vital to understanding directional quenching. In addition, the coarsening processes of the lamellar and the columnar pattern have been unclear yet. Here we investigate the dynamics and mechanisms behind columnar pattern formation and the coarsening process during directional quenching using a two-dimensional numerical simulation. 


\section{METHODS}

The dynamics of phase separation under homogeneous quenching is often studied using the Cahn-Hilliard-Cook equation $[1,4,34]$. For directional quenching, we used a modified Cahn-Hilliard-Cook equation proposed in Ref. [35]. This equation includes the concentration transport due to the temperature gradient (Ludwig-Soret effect) [36]. Here we assume that the shallow quenching, that is, the phase separation occurs near the critical temperature. In such a case, the temperature gradient terms can be considered negligible [32]. We normalize the length and the time using the correlation length and a characteristic time at an initial temperature, respectively; we normalize the concentration $\phi$ using the concentration after phase separation. The normalized equations are given as

$$
\frac{\partial \phi}{\partial t}=\nabla^{2}\left[\epsilon(x, t) \phi+\phi^{3}-\nabla^{2} \phi\right]-\nabla \cdot \vec{g},
$$

where $\phi, t, \epsilon$, and $\vec{g}$ are normalized concentration, normalized time, normalized temperature, and a random flux, respectively. When $\epsilon$ is constant, $\epsilon$ should be 1 or -1 in the normalized equation. We note that the sign of $\epsilon$ changes by the temperature in realistic phase separations. When $\epsilon=1$, the mixed state is stable; when $\epsilon=-1$, phase separation occurs. Assuming local equilibrium, $\vec{g}$ obeys the fluctuationdissipation relation. For the dimensionless noise in these equations, it is

$$
\left\langle\vec{g}_{i}(\vec{r}, t) \vec{g}_{j}\left(\vec{r}^{\prime}, t^{\prime}\right)\right\rangle=\Theta(\vec{r}) \delta_{i j} \delta\left(\vec{r}-\vec{r}^{\prime}\right) \delta\left(t-t^{\prime}\right),
$$

where $i, j=x, y$ and $\Theta$ corresponds to fluctuation strength. The role of the thermal fluctuations will be discussed under Discussion. Figure 1 shows the phase diagram for our system with respect to $\phi$ and $\epsilon$. When $|\phi|<\phi_{\mathrm{SD}}(=1 / \sqrt{3})$, i.e., inside the dashed line in Fig. 1, the system is unstable, i.e., thermal fluctuations grow with time (spinodal decomposition). On the other hand, when $|\phi|>\phi_{\mathrm{SD}}$ between the dashed line and the solid line in Fig. 1, the system is metastable; phase separation occurs via a nucleation and growth mechanism. Here we set the initial state to be asymmetric in composition, with a mean concentration $\bar{\phi}=0.1$.

We prepared an initial concentration field by annealing the whole system at $\epsilon=1$ before a directional quench. In the directional quench, $\epsilon(x, t)$ varies over both time and space as follows:

$$
\epsilon(x, t)=\left\{\begin{array}{ll}
1 & x>V t \\
-1 & x \leqslant V t
\end{array},\right.
$$

where $V$ is the velocity of the quenching front and $t$ is time. We set $\Theta(\vec{r})=0.001$ for all $\vec{r}$. We used periodic boundary conditions in the $y$ plane and set a free surface in the $x$ plane. No surface field acts on the mixture, unlike the problem of surface-directed spinodal decomposition [13-19]. Here we start the directional quench from the left surface at a constant velocity $V$ at $t=0$. The simulation grid size was $x$ : $y=400: 2000$. We neglect hydrodynamics. We performed this simulation seven times starting from the different initial concentration field. The data and the error to be shown are the mean and the standard deviation of the seven simulations.

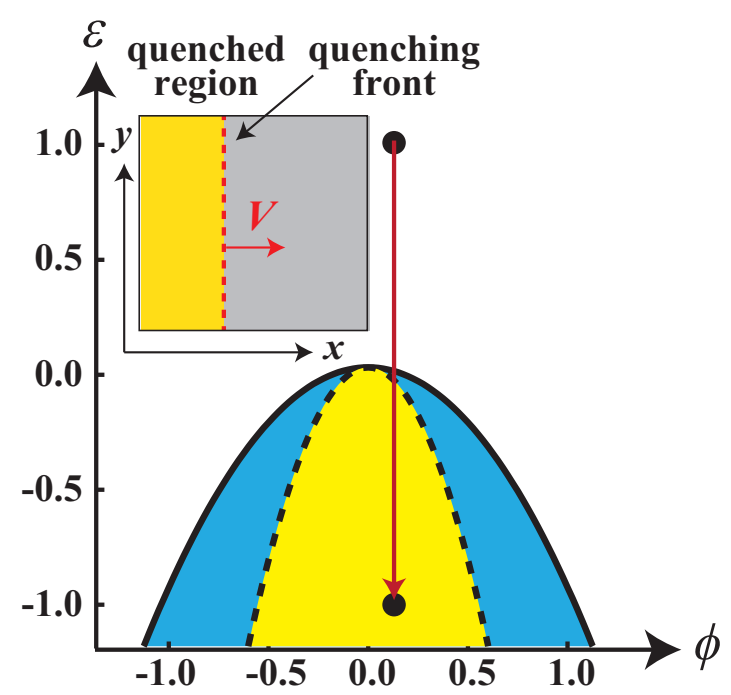

FIG. 1. Phase diagram with respect to $\phi$ and $\epsilon$. When $\epsilon$ is negative, phase separation occurs. When $|\phi|<\phi_{\mathrm{SD}}(=1 / \sqrt{3})$, the system is unstable, and thermal fluctuations grow with time. When $|\phi|>\phi_{\mathrm{SD}}$, the system is metastable. We set the initial state such that the mean concentration $\bar{\phi}$ is 0.1 . We quench the system using a front moving in the direction given by the red arrow. $\epsilon$ is switched from 1 to -1 when the front passes. Inset: System coordinates ( $x$ and $y$ axes) for the directional quench. The yellow region is the quenched region; the dashed line corresponds to the quenching front which moves at a constant velocity $V$.

\section{RESULTS}

\section{A. Pattern diagram}

First, we study the $V$ dependence of the pattern formed during phase separation with directional quenching. Figure 2(a) shows how the phase separation pattern changes over time when $V=100$ and $t=50$ [Fig. 2(a1)] and 5000 [Fig. 2(a2)]. The whole system becomes quenched by $t<4$. We find that a random droplet pattern is formed, just like for homogeneous quenching. This is to be expected when the dynamics of the phase separation is much slower than the migration of the quenching front [see Fig. 2(a1)]. The pattern formed changes significantly when $V$ is small. Figure 2(b) shows how the pattern changes over time when $V=0.07$ at $t=250$ [Fig. 2(b1)] and 10000 [Fig. 2(b2)]. Dashed lines show the locations of the quenching front. At an early stage, the majority phase appears as a layer near $x=0$, since $\bar{\phi}$ is asymmetric. Next, the minority phase appears as a layer parallel to the first majority phase. Finally, a lamellar pattern is formed parallel to the quenching front. However, when $V<0.07$, a columnar pattern, i.e., layers perpendicular to the quenching front is formed instead of lamellae. Figure 2(c) shows the time evolution of the phase separation pattern when $V=0.03$ at $t=$ 1000 [Fig. 2(c1)] and 15000 [Fig. 2(c2)]. Initially, a layer of the majority phase forms near the starting line in the same way. However, now the minority phase emerges perpendicular to the first layer [see Fig. 2(c1)]. The columnar pattern then continues to grow following the migration of the front [see Fig. 2(c2)]. Figure 2(d) summarizes the patterns formed for different $V$. The symbols correspond to the points simulated. When $V \geqslant 2$, we find that a droplet pattern is formed. When 

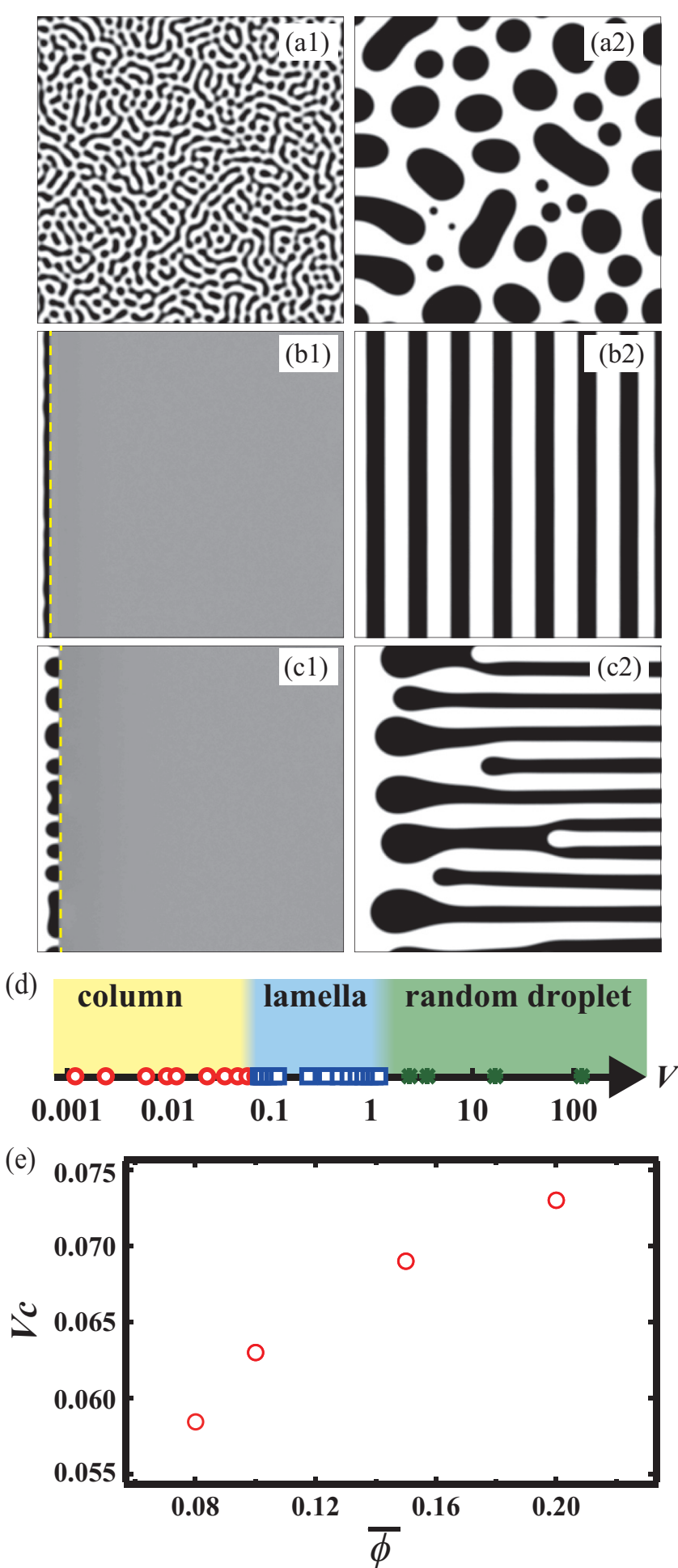

FIG. 2. Pattern formation induced by directional quenching. Each image is a small $400 \times 400$ section of the whole image. (a) $V=100$, at $t=50$ (a1) and 5000 (a2). A random droplet pattern is formed. (b) $V=0.07$, at $t=250$ (b1) and 10000 (b2). A lamellar pattern is formed. (c) $V=0.03$, at $t=1000$ (c1) and 15000 (c2). A columnar pattern is formed. Dashed lines in (b1) and (c1) represent the quenching front. (d) Pattern diagram with respect to $V$. Symbols correspond to the points simulated. The circle, square, and cross correspond to columnar, lamellar, and random droplet patterns, respectively. (e) The transition velocity $V_{c}$ as a function of $\bar{\phi} . V_{c}$ increases with increasing $\bar{\phi}$.
$0.07 \leqslant V<2$, a lamellar pattern is formed, and when $V<$ 0.07 , a columnar pattern is formed. These are qualitatively consistent with previous findings [22]. Here we stress that the mechanism by which this transition between lamellar and columnar patterns occurs is not yet understood.

In addition, we also investigate the transition velocity $V_{c}$ as a function of $\bar{\phi}$ shown as in Fig. 2(e). We find that $V_{c}$ increases with increasing $\bar{\phi}$. This reason will be discussed later. We also note that the first majority phase is not formed when $\bar{\phi}$ is close to 0 since both phases are almost symmetric. Then, it is found that the lamellar pattern is not observed for $\bar{\phi} \leqslant 0.05$.

\section{B. Instability of the interface}

We study how a lamellar pattern is formed when $V=0.07$ in more detail. This is close to the speed at which a columnar pattern is seen instead [see Fig. 2(d)]. At the beginning, a layer of the majority phase emerges and grows with a flat interface. The interface fluctuates as shown in Fig. 3(a), a horizontally stretched image at $t=200(V=0.07)$. To evaluate the fluctuations quantitatively, we define $\sigma(x, t)$ as $\sigma(x, t)=$ $\int d y\left(\phi(x, y, t)-\langle\phi(x, y, t)\rangle_{y}\right)^{2} / L_{y}$, where $\langle\phi(x, y, t)\rangle_{y}$ is the mean concentration averaged over $y$ at fixed $x$ and $t$, and $L_{y}$ is the simulation box size in the $y$ direction. We also compute $\sigma\left(x_{m}, t\right)$, where $x_{m}$ is where $\sigma(x, t)$ becomes maximum at fixed $t$. Here we confirm that $x_{m}$ corresponds to the position of the interface of the layer at the front of the growth. Figure 3(b) shows the time evolution of $\sigma\left(x_{m}, t\right)$. We find that $\sigma\left(x_{m}, t\right)$ suddenly increases at $t=100$ as the interface begins to fluctuate. Next, we see a minority layer appear parallel to the first majority layer. $\sigma\left(x_{m}, t\right)$ shows a maximum at $t=200$ when this layer forms. Subsequently, $\sigma\left(x_{m}, t\right)$ decreases for $t>200$ as the fluctuations diminish to decrease the interfacial energy. Note that when a minority layer appears next to the first majority layer, $\sigma\left(x_{m}, t\right)$ shows a large peak (blue arrow); on the other hand, when the next majority layer appears, $\sigma\left(x_{m}, t\right)$ exhibits only a small peak (red arrow). The reason for this will be discussed later, Next, we compute $\sigma\left(x_{m}, \tau\right)$, where $\tau$ is when $\sigma\left(x_{m}, t\right)$ shows a local maximum. Figure 3(c) shows $\sigma\left(x_{m}, \tau\right)$ as a function of $V$. We find that $\sigma\left(x_{m}, \tau\right)$ is larger for smaller $V$, that is, the fluctuations become larger with decreasing $V$. When $V<0.07$, we find that the fluctuations grow large enough such that the phase separation occurs perpendicular to the interface, parallel to the fluctuations, rather than the fluctuation being suppressed [see Fig. 2(c1)]. The pattern continues to grow in the $x$ direction, and a columnar pattern is formed.

In addition, we also investigate the relationship between the time evolution of $\langle\phi(x, y, t)\rangle_{y}$ and the position of the quenching front. Figure 4 shows the minimum position of $\langle\phi(x, y, t)\rangle_{y}$ (circle) and the position of the quenching front (triangle) when $V=0.07$. We also calculate $\Delta \xi$, which is the distance between the minimum position of $\langle\phi(x, y, t)\rangle_{y}$ and the position of the quenching front. $\Delta \xi$ is negative and constant for $t<100$, and rapidly increases when $t \geqslant 100$, This means that for when $t \geqslant 100$, the interface of the first layer is located inside the region where the quenching front has passed and thus that the region near the interface is undergoing spinodal decomposition [see Fig. 3(a)]. Here we note that the time when $\Delta \xi$ increases is the same as when 

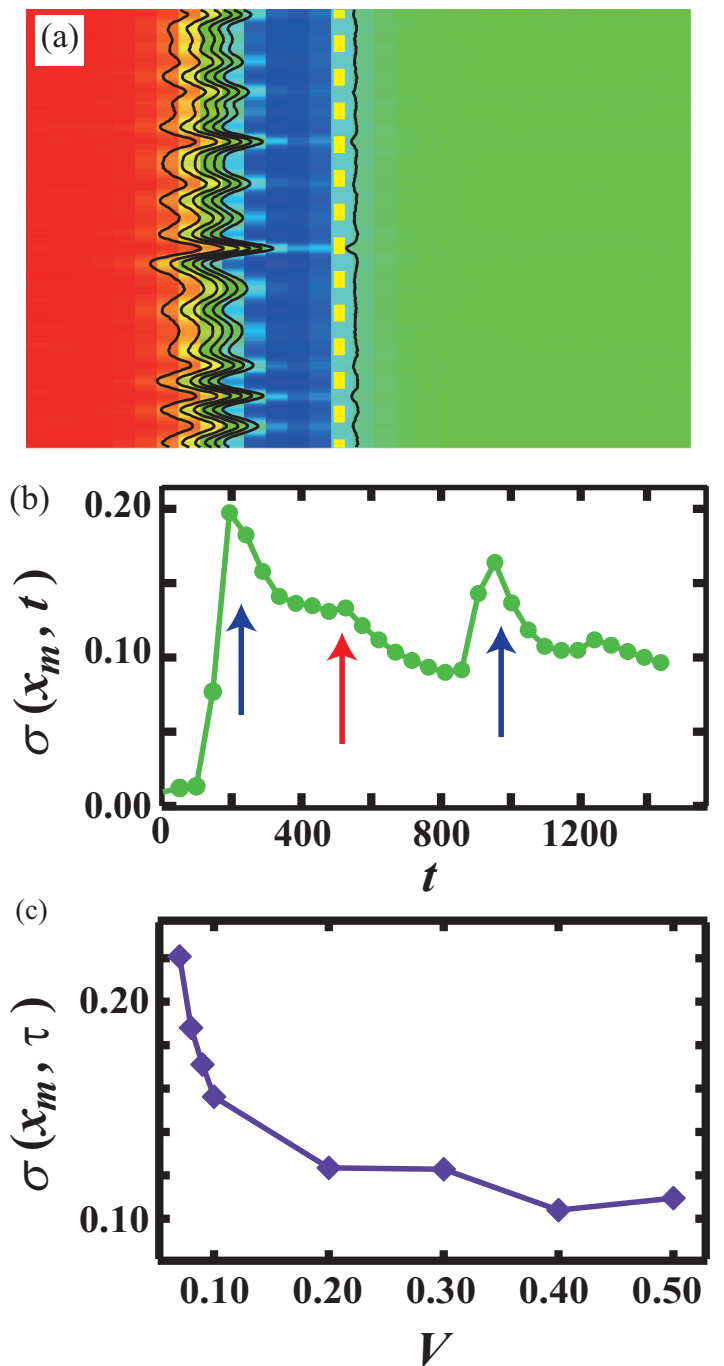

FIG. 3. (a) The concentration field when $V=0.07$ at $t=200$. The image is stretched in the $x$ direction for clarity; the region shown is a $400 \times 30$ area. Solid lines represent contours for the concentration field. The dashed line represents the quenching front. The interface fluctuates. (b) $\sigma\left(x_{m}, t\right)$ as a function of time, where $x_{m}$ is the $x$ coordinate at fixed $t$ where the largest fluctuations are seen. Red arrows signify the appearance of a minority layer; blue arrows show when a majority layer emerges. (c) $\sigma\left(x_{m}, \tau\right)$ as a function of $V$, where $\tau$ is the time when $\sigma\left(x_{m}, t\right)$ is maximum. This suggests that the fluctuations become larger with decreasing $V$. The errors are smaller than the symbol size.

$\sigma\left(x_{m}, t\right)$ increases in Fig. 3(b). The interface starts to fluctuate when the interface is in the spinodal decomposition regime. Thus, the instability of the interface is related to spinodal decomposition.

We also consider the effect of flux. We may compute the flux in the $x$ and $y$ directions, $j_{x}$ and $j_{y}$, using $j_{i}=\nabla_{i}[\epsilon \phi+$ $\phi^{3}-\nabla^{2} \phi$ ], where $i=x, y$. Figures 5(a) and 5(b) show $j_{x}$ and $j_{y}$ at $x=x_{m}$ and $t=\tau$ when $V=0.5$ and 0.07 , respectively. A moving average filter is applied to remove noise. We find that $\left\langle j_{x}\right\rangle_{y}$ at $V=0.07$ is much smaller than that at $V=0.5$. Averaged over $y$ and given as a function of $V$, we find that $\left\langle j_{x}\right\rangle_{y}$ decreases with decreasing $V$ as shown in Fig. 5(c). This is mirrored by how $\Delta \xi$ varies with $V$ as shown in Fig. $5(\mathrm{~d})$. We

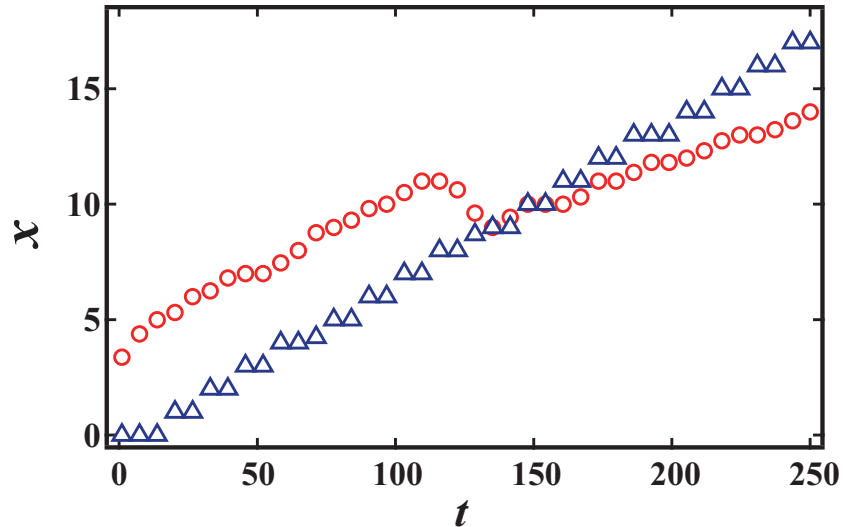

FIG. 4. Position of the quenching front (triangles) and the minimum of $\phi$ (circle) at $V=0.07$. The minimum of $\phi$ is beyond the quenching front for $t<100$. For $t>100$, the quenching front passes the $\phi$ minimum. This means that the layer growth cannot follow the migration of the quenching front for $t>100$. This is almost the same time as when $\sigma\left(x_{m}, t\right)$ increases [see Fig. 3(b)]. The errors are smaller than the symbol.

see that the close relationship between $\Delta \xi$ and $\left\langle j_{x}\right\rangle_{y}$ derives from the action of the quenching front on the flow field.

In the case of directional quenching, there is a large initial flux in the direction in which the front is propagating, leading to the formation of a layer. When $\left\langle j_{x}\right\rangle_{y}$ is large, $\langle\phi(x, y, t)\rangle_{y}$ rapidly decreases; when $\left\langle j_{x}\right\rangle_{y}=0,\langle\phi(x, y, t)\rangle_{y}$ is conserved. Initially, in the presence of the flux, $\langle\phi(x, y, t)\rangle_{y}$ steadily decreases. While $-\phi_{\mathrm{SD}}<\phi<\phi_{\mathrm{SD}}$, the system may undergo spinodal decomposition, and fluctuations may grow. Finally, the concentration becomes lower than $-\phi_{\mathrm{SD}}$, and the region enters a nucleation and growth regime (see Fig. 1), allowing for the nucleation of droplets. Importantly, whether droplets will be nucleated or not depends on whether the fluctuations have grown large enough by the time the nucleation and growth regime sets in. Thus, the key parameter becomes $t_{\text {int }}$, the time taken until the condition $\langle\phi(x, y, t)\rangle_{y}<-\phi_{\mathrm{SD}}$ is met;
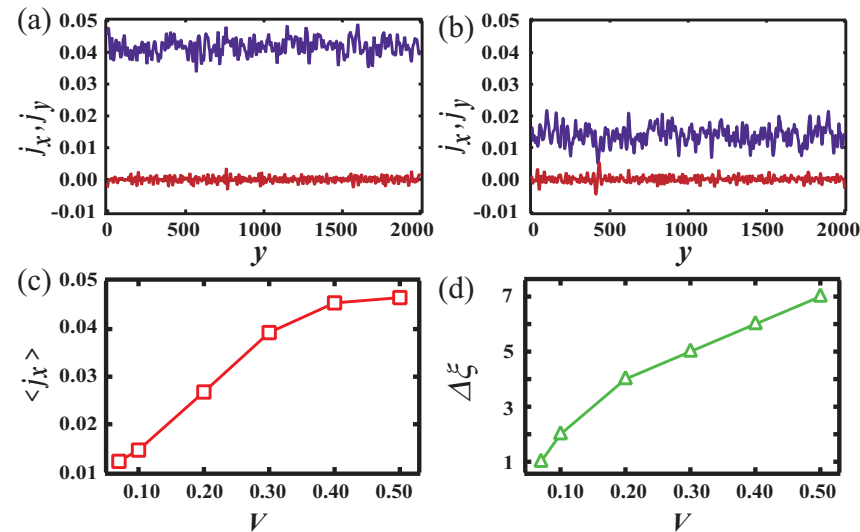

FIG. 5. $j_{x}$ (blue line) and $j_{y}$ (red line) as a function of $y$ at $x=x_{m}$ and $t=\tau$ when (a) $V=0.5$ and (b) $V=0.07$. A moving average is taken. (c) $V$ dependence of $\left\langle j_{x}\right\rangle$ averaged over $y$. The flux in the $x$ direction decreases with decreasing $V$. (d) $\Delta \xi(\tau)$ as a function of $V$. For larger $V, \Delta \xi(\tau)$ becomes large. The errors are smaller than the symbol. 


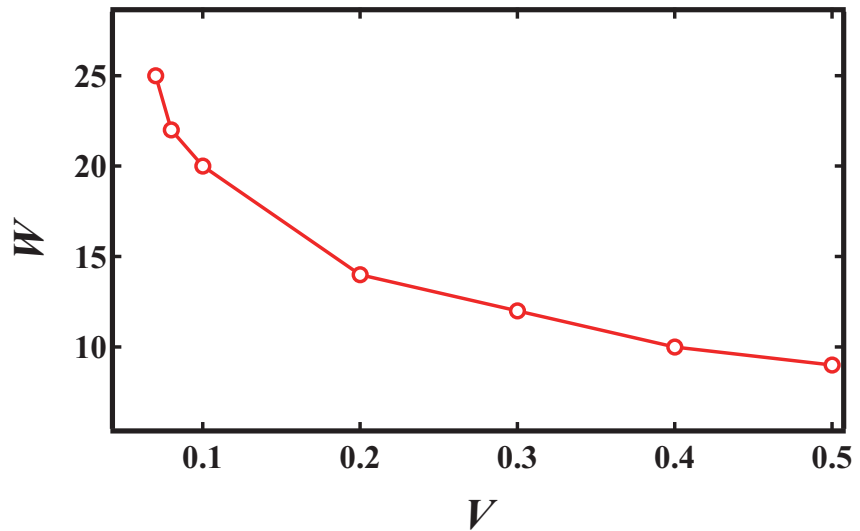

FIG. 6. The steady width $W$ of the lamellar as a function of $V$. $W$ increases with decreasing $V$. The errors are smaller than the symbol.

this is the key distinction between the formation of lamellar and columnar patterns. For larger $V,\left\langle j_{x}\right\rangle_{y}$ is large. Thus, $t_{\text {int }}$ is too short for fluctuations to grow, as shown in Fig. 3(c). Meanwhile, for smaller $V,\left\langle j_{x}\right\rangle_{y}$ is suppressed and $t_{\text {int }}$ becomes larger. Thus, when $V<0.07$, we see that fluctuations due to spinodal decomposition are able to grow large enough to nucleate droplets of the minority phase, allowing a columnar pattern to form.

\section{Coarsening of the patterns}

Then we investigate the coarsening of the lamellar pattern. We find that the lamellar pattern is stable and stationary with time in this simulation. It is natural that the interface of the lamellar is flat and the coarsening is induced by the curvature of the interface. Figure 6 shows the steady width $W$ of the lamellar as a function of $V$. When the growth of the first majority layer cannot follow the migration of the quenching front, the minority layer appears. Thus $W$ should be smaller with larger $V$ shown as in Fig. 6.

We also investigate the coarsening of the columnar pattern. First, we compute the most unstable wavelength $\lambda$ at the interface of the first layer. We calculate Fourier transform of $\phi$ at $x=x_{m}$ at the onset time of the increase of $\sigma\left(x_{m}, t\right)$ and $\lambda$ is determined from its peak. Figure 7 shows the $V$ dependence

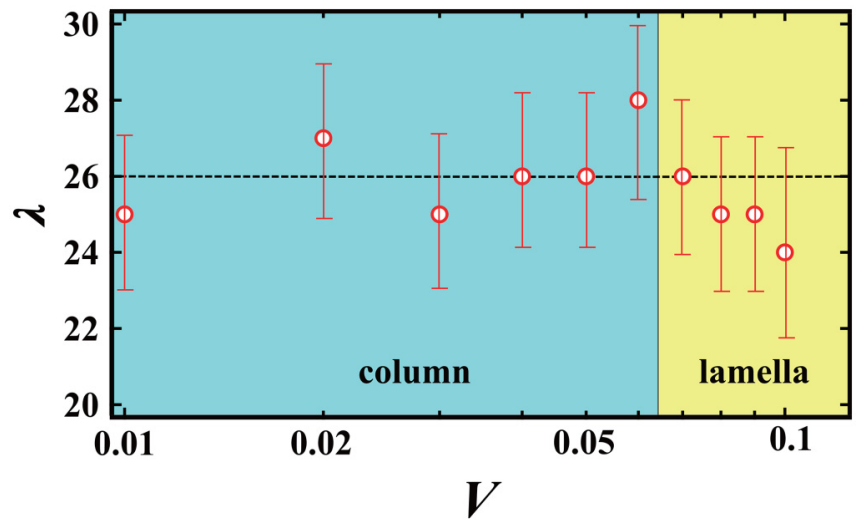

FIG. 7. $\lambda$ as a function of $V . \lambda$ is almost constant for $V<0.1$, regardless of whether the formed pattern is columnar or lamellar.
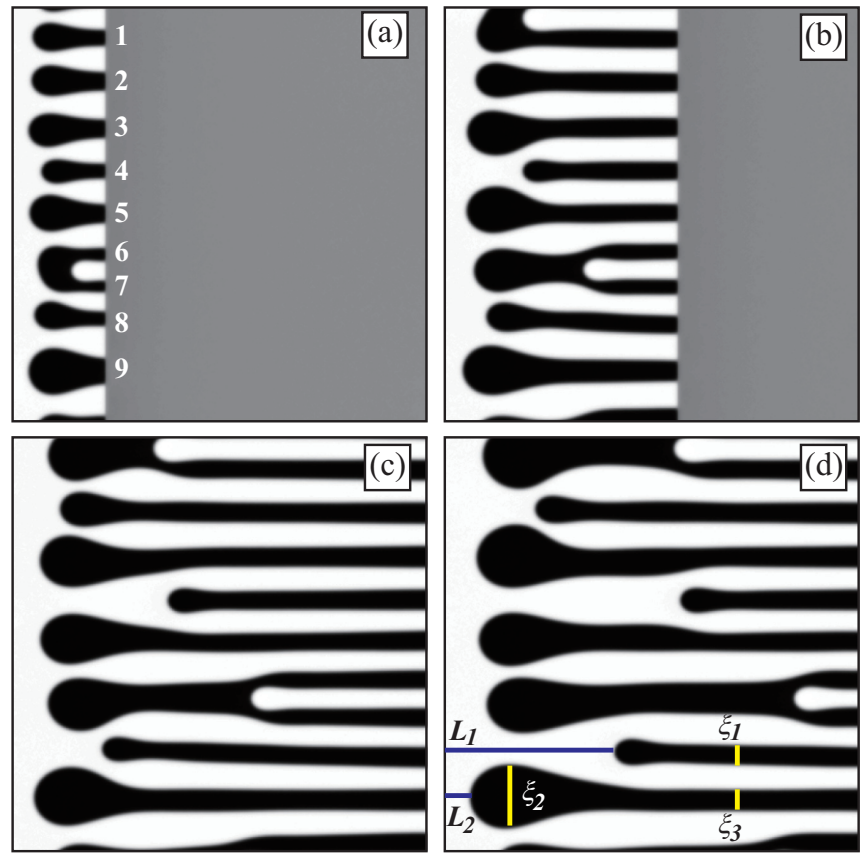

FIG. 8. Elongation process of the column at $V=0.03$. Each image is a small $400 \times 400$ section of the whole image; $t=$ (a) 3000 , (b) 7500 , (c) 13500 , and (d) 21000 . We put the number to the columns shown as in (a). We investigate time evolutions of the five typical lengths, $\xi_{1}, \xi_{2}, \xi_{3}, L_{1}$, and $L_{2}$ shown as in (d).

of $\lambda$. We find that $\lambda$ is almost constant for $V<0.1$, regardless of whether the formed pattern is columnar or lamellar. Here we note that $\lambda$ is not equal to the most unstable wavelength in the homogeneous quenching system. It is found that analytical prediction of $\lambda$ by a linear instability analysis is difficult in the directional quenching system since $\phi$ is far from a steady state when the interface become unstable. Although this is an interesting theoretical work, it is beyond the scope of this research.

Next, we investigate how the initial columns emerge. The instability of the interface can be observed even in the lamellar regime of $V$ shown as in Fig. 3(a). Those fluctuations decay with the migration of the quenching front. In the columnar regime, the columns can elongate from some of large fluctuated points, on the other hand, the other small fluctuations decay with time as same as the lamella regime. In addition, some of the columns quickly coalesce if the distance between the nearest columns is small. Thus, it is found that the period of the columns is not equal to $\lambda$.

Finally, we show the elongation process of the column at the later stage. Figure 8 show the elongation process of the columns. Each image is a small $400 \times 400$ section of the whole image. At $t=3000$, nine columns of the minority phase are observed, shown as in Fig. 8(a). It is found that the edges of the sixth and the seventh columns are coalesced. During the elongation process, the coalescence of the the sixth and the seventh is proceeding and the majority column between them moves shown as in Fig. 8(b). It is also found that the fourth and the eighth of the minority column also moves to right. Then we quantitatively investigate time evolutions of the width of the active column $\xi_{1}$ and the distance of the active 


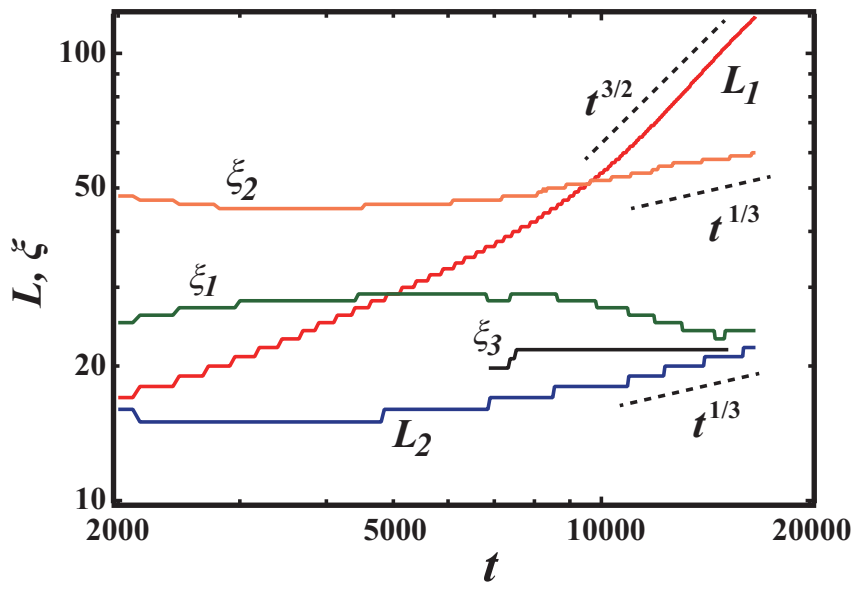

FIG. 9. Time evolutions of the five typical lengths, $\xi_{1}, \xi_{2}, \xi_{3}, L_{1}$, and $L_{2} . \xi_{1}$ is constant and $L_{1}$ increases with $t^{3 / 2}$ at the later stage. Meanwhile, $\xi_{2}$ and $L_{2}$ increases with $t^{1 / 3}$ at the later stage and $\xi_{3}$ is almost constant.

column $L_{1}$. It is found that $\xi_{1}$ is constant with time shown as in Fig. 9, and thus those active columns move to the right without changing their shapes. We also find that $L_{1}$ increases with $t^{3 / 2}$ at the later stage and that this zipperlike motion is faster than the usual coarsening. Unfortunately, the theoretical explanation for the exponent $3 / 2$ is unclear and it is a future work. Meanwhile, the other minority columns remain at the similar position [Figs. 8(c) and 8(d)]. We also investigate time evolutions of the typical lengths, the edge size of the static column $\xi_{2}$, the width of the static column $\xi_{3}$, and the distance of the static column $L_{2}$. Figure 9 shows the time evolutions of $\xi_{2}, \xi_{3}$, and $L_{2}$. It is found that $\xi_{2}$ and $L_{2}$ increase with $t^{1 / 3}$ at the later stage. This exponent is same as the coarsening in the homogeneous quenching system. $\xi_{3}$ is almost constant since the interface is flat. Thus, the typical length scales of the columnar pattern depends on the time and the space due to the combination of the active and the static columns. Here it is found that the edge size of the column plays an important role for the elongation. If the edge is smaller than that of the neighbor column, then the column moves to the quenching front. On the other hand, the column with the large edge stays at the same position and the edge becomes larger. We confirm that those time evolutions are almost same if we choose different columns.

\section{DISCUSSION}

Here we discuss the reasons why the flux in $x$ direction is suppressed when $V$ is small. At the front, we expect a homogeneous state to preferred, i.e., the concentration is $\bar{\phi}$. The flux is also expected to be zero. In other words, the front acts as a confining boundary for the flow field. With this picture, we may estimate the $|\nabla \phi|^{2}$ term in the free energy using a simple linear approximation, $\left[\left(\bar{\phi}-\phi_{m}\right) / \Delta \xi\right]^{2}$, where $\phi_{m}$ is the minimum concentration at the position indicated by $\Delta \xi$. When $\Delta \xi$ is large, the only way to decrease the energy is to have a lower $\phi_{m}$. This leads to a greater flux due to a larger concentration gradient. Conversely, when $\Delta \xi$ is small, the flux is small. Since $\Delta \xi$ is related with $V$ shown as in Fig. 5(d), the flux is suppressed when $V$ is small. This suppression due to effective confinement is in agreement with our previous work [33].

Then we also consider $\bar{\phi}$ dependence of $V_{c}$ with the effective confinement. As we described above, $|\nabla \phi|^{2}$ term in the free energy positively depends on $\bar{\phi}$. When $\bar{\phi}$ is large, the effective confinement effect is strong and then the flux in $x$ direction is suppressed. Thus $V_{c}$ increases with increasing $\bar{\phi}$ shown as in Fig. 2(e). In addition, we note that since $\bar{\phi}$ is positive, the confinement effect is stronger when a minority layer emerges than for a majority layer. This explains why peaks in $\sigma\left(x_{m}, t\right)$ are large when a layer of the minority phase appears; note the blue arrows in Fig. 3(b). On the other hand, the peaks are small when a minority layer emerges; note the red arrow in Fig. 3(b).

Finally, we discuss the roles of the thermal fluctuations. In general, the thermal fluctuations have mainly two effects. One is a trigger for the phase separation. If $\phi$ is completely homogeneous, then the phase separation never occurs [see Eq. (1)]. The other is for the coarsening process at the later stage. The thermal fluctuations can impose the curvature to a flat interface and the curvature drives the coarsening. In our simulation, it is found that the columnar pattern cannot be formed when we set $\Theta=0$ after $t=0$. Thus, the thermal fluctuations are a trigger not only for the phase separation but also for the spinodal decomposition at the interface of the lamellar. Meanwhile, we found that the width of the lamellar $W$ is steady at the later stage. It means that the thermal fluctuations do not affect the coarsening at the later stage.

\section{SUMMARY}

We investigated the mechanism behind the formation of columnar patterns during directional quenching. We find that the interface of the first layer composed of the majority component fluctuates when the layer growth cannot follow the movement of the quenching front. We confirm that the origin of this fluctuation is instability in the system due to spinodal decomposition. It is also found that the quenching front acts as an effective boundary, and plays an important role for the suppression of the flux. The flux in the $x$ direction is strongly suppressed when $V$ is small, while it is less suppressed for larger $V$. For small $V$, this suppression gives enough time for fluctuations to grow. When $V<0.07$, droplets of the minority phase can nucleate at the interface, resulting in a phase separation that creates a columnar phase. We also investigate the coarsening of the patterns. The lamellar pattern is steady since the interface is flat, while the columnar pattern shows unique coarsening. It is found that the columns with small edge actively move, on the other hand, the columns with large edge stays at the same position. Our study not only reveals the mechanism behind columnar pattern formation but also shows that the quenching front has a confinement effect. These results apply generally to phase separating mixtures and proposes a well-characterized mechanism for nonequilibrium phenomena in inhomogeneous systems.

\section{ACKNOWLEDGMENTS}

R.K. was supported by JSPS KAKENHI (17H02945). 
[1] J. W. Cahn and J. E. Hilliard, J. Chem. Phys. 28, 258 (1958).

[2] Y. Oono and S. Puri, Phys. Rev. Lett. 58, 836 (1987).

[3] S. Puri, Phys. Lett. A 134, 205 (1988).

[4] A. Onuki, Phase Transition Dynamics (Cambridge University Press, Cambridge, UK, 2002).

[5] H. Tanaka and T. Araki, Phys. Rev. Lett. 85, 1338 (2000).

[6] H. Tanaka, T. Araki, T. Koyama, and Y. Nishikawa, J. Phys.: Condens. Matter 17, S3195 (2005).

[7] I. W. Hamley, Introduction to Soft Matter: Synthetic and Biological Self-Assembling Materials (Wiley, Hoboken, NJ, 2007).

[8] M. T. Connor, S. Roy, T. A. Ezquerra, and F. J. Balta Calleja, Phys. Rev. B 57, 2286 (1998).

[9] R. C. Ball and R. L. H. Essery, J. Phys.: Condens. Matter 2, 10303 (1990).

[10] L. Berthier, Phys. Rev. E 63, 051503 (2001).

[11] A. A. Golovin, A. A. Nepomnyashchy, S. H. Davis, and M. A. Zaks, Phys. Rev. Lett. 86, 1550 (2001).

[12] P. C. Hohenberg and B. I. Halperin, Rev. Mod. Phys. 49, 435 (1977).

[13] S. Puri and K. Binder, Phys. Rev. A 46, R4487 (1992).

[14] S. Puri and H. L. Frisch, J. Phys.: Condens. Matter 9, 2109 (1997).

[15] L. Kielhorn and M. Muthukumar, J. Chem. Phys. 111, 2259 (1999).

[16] H. Tanaka and T. Araki, Europhys. Lett. 51, 154 (2000).

[17] L.-T. Yan, J. Li, Y. Li, and X.-M. Xie, Macromolecules 41, 3605 (2008).
[18] A. Krekhov, V. Weith, and W. Zimmermann, Phys. Rev. E 88, 040302(R) (2013).

[19] Y. Iwashita and Y. Kimura, Soft Matter 9, 10694 (2013).

[20] A. A. Kulkarni, J. Kohanek, K. I. Tyler, E. Hanson, D.-U. Kim, K. Thornton, and P. V. Braun, Adv. Opt. Mater. 6, 1800071 (2018).

[21] R. Shimizu and H. Tanaka, Sci. Adv. 3, eaap9570 (2017).

[22] H. Furukawa, Physica A 180, 128 (1992).

[23] H. Furukawa, J. Phys. Soc. Jpn. 63, 3744 (1994).

[24] B. Liu, H. Zhang, and Y. Yang, J. Chem. Phys. 113, 719 (2000).

[25] A. Krekhov, Phys. Rev. E 79, 035302(R) (2009).

[26] N. Oikawa and R. Kurita, Sci. Rep. 6, 28960 (2016).

[27] R. Kurita, Sci. Rep. 7, 6912 (2017).

[28] K. Morinaga, N. Oikawa, and R. Kurita, Sci. Rep. 8, 12503 (2018).

[29] S. Roy, S. Dietrich, and A. Maciolek, Phys. Rev. E 97, 042603 (2018).

[30] S. Roy and A. Maciolek, Soft Matter 14, 9326 (2018).

[31] K. Zhang, Q. Zhang, H. Zhang, J. Shen, Q. Niu, and R. Xia, Macromol. Chem. Phys. 219, 1700527 (2018).

[32] T. Tsukada and R. Kurita, J. Phys. Soc. Jpn. 88, 044603 (2019).

[33] T. Tsukada and R. Kurita, Sci. Rep. 9, 15764 (2019).

[34] H. E. Cook, Acta Metall. 18, 297 (1970).

[35] P. K. Jaiswal, S. Puri, and K. Binder, Europhys. Lett. 103, 66003 (2013).

[36] J. K. Platten, J. Appl. Mech. 73, 5 (2005). 